\title{
Protecting the Health and Well-being of Populations from Disasters: Health and Health Care in The Sendai Framework for Disaster Risk Reduction 2015-2030
}

\author{
Amina Aitsi-Selmi, MBBChir, MA(cantab), MRCP, MPH, MFPH, PhD; ${ }^{1,2}$ \\ Virginia Murray, FRCP, FRCPath, FFPH, FFOM ${ }^{1,3}$
}

1. International Public Health, Public Health England, London, United Kingdom

2. Research Department of Epidemiology and Public Health, University College London, London, United Kingdom

3. United Nations Office for Disaster Risk Reduction (UNISDR) Scientific \& Technical Advisory Group, Geneva, Switzerland

\section{Correspondence:}

Amina Aitsi-Selmi, MBBChir, MA(cantab), MRCP, MPH, MFPH, PhD

Global Disaster Risk Reduction/ International Public Health

Public Health England

Wellington House

133-155, Waterloo Road

London, SE1 8UG, United Kingdom

E-mail: amina.aitsi-selmi@phe.gov.uk

\section{Conflicts of interest: none}

Keywords: disaster; disaster risk reduction; emergency response; global health policy; public health

\section{Abbreviations:}

DRR: Disaster Risk Reduction

STAG: Scientific and Technical Advisory Group

UN: United Nations

UNISDR: United Nations Office for Disaster Risk Reduction

WADEM: World Association for Disaster and Emergency Medicine

WHO: World Health Organization

Received: August 17, 2015

Accepted: September 7, 2015

Online publication: December 17, 2015

doi:10.1017/S1049023X15005531

\section{Abstract:}

The Sendai Framework for Disaster Risk Reduction (DRR) 2015-2030 is the first of three United Nations (UN) landmark agreements this year (the other two being the Sustainable Development Goals due in September 2015 and the climate change agreements due in December 2015). It represents a step in the direction of global policy coherence with explicit reference to health, economic development, and climate change. The multiple efforts of the health community in the policy development process, including campaigning for safe schools and hospitals, helped to put people's mental and physical health, resilience, and well-being higher up the DRR agenda compared with its predecessor, the 2005 Hyogo Framework for Action. This report reflects on these policy developments and their implications and reviews the range of health impacts from disasters; summarizes the widened remit of DRR in the post-2015 world; and finally, presents the science and health calls of the Sendai Framework to be implemented over the next 15 years to reduce disaster losses in lives and livelihoods.

Aitsi-Selmi A, Murray V. Protecting the health and well-being of populations from disasters: health and health care in The Sendai Framework for Disaster Risk Reduction 2015-2030. Prehosp Disaster Med. 2016;31(1):74-78.

\section{Introduction}

The 2004 Indian Ocean earthquake and tsunami illustrated how natural hazards can become devastating when social vulnerability is high. ${ }^{1}$ Similarly, the severe 2011 floods in Thailand that affected the car industry in Japan and the global computer industry for a significant period of time showed how interdependence can promote development but increase vulnerability. ${ }^{2}$ Such events have urged the global community to identify global priorities for action and practical steps that are required to achieve disaster resilience as an integral part of economic activity; for example, in adopting the Hyogo Framework of Action, ${ }^{3}$ but also at local or national levels to improve the emergency management of disasters. ${ }^{4}$

For reasons that are not understood fully, disasters are growing in frequency and in impact, ${ }^{5}$ but there is global recognition that disasters are not natural events - they are the product of the interaction between hazards and socially constructed vulnerabilities and exposures. In parallel, an expansion of perspective on what constitutes a hazard has also occurred, with extreme weather events related to climate change becoming a driver for adaptation and mitigation. ${ }^{6}$ In recognition of the changing nature of vulnerability and the amplification of the risk from natural hazards by human activity, threats related to rapid and unmanaged urbanization and globalization have also entered the disaster risk reduction (DRR) lexicon, alongside other advances in risk assessment to include social vulnerability and capacity to respond. ${ }^{7}$

The expansion of DRR to include risk assessments addressing vulnerability and capacity has been compared to the widening of health activities to include prevention and becoming increasingly concerned with the total health system and not only the eradication of a particular disease affecting an individual patient. ${ }^{8}$ The Sendai Framework for Disaster 
Risk Reduction 2015-2030 was born of the need to ensure DRR policy reflects this evolved understanding of the complexity of disaster risk in the $21^{\text {st }}$ century and builds in health as a key outcome for the next 15 years. ${ }^{9}$ The implementation of the Sendai Framework calls for a multi-sectoral, transdisciplinary approach supporting closer collaboration among relevant actors to prevent, prepare for, and recover from disasters, as well as respond effectively. ${ }^{6}$

This report reviews the elements of the new Sendai Framework that call on the health and scientific community to take action to help reduce disaster impacts through prevention, preparedness, response, recovery, and rehabilitation and provides some examples of good practice.

\section{Report}

Health, Health Care, and Disasters

The consequences of disasters on health and well-being are many. In most disasters, deaths tend to occur from blunt trauma; for example, when buildings, including hospitals, collapse. ${ }^{10}$ Risks can be compounded when population displacement and overcrowding in evacuation or re-housing facilities leads to a further increase in the risk of infectious disease outbreaks, or when overwhelmed medical services are unable to deliver on elective functions, such as chronic disease management, putting those who need life-saving medication, such as insulin for diabetes, in a particularly vulnerable position. ${ }^{11}$ For example, during Typhoon Haiyan in the Philippines, the major medical and public health needs of the affected people were not injury-related, but they were the result of a lack of measures to prevent infectious diseases and the worsening of non-communicable diseases due to the lack of access to food, water, housing, and medicine. ${ }^{12}$

Furthermore, the mental health consequences of traumatic incidents, in general, can be prolonged with stress to people, families, and communities resulting in short-term fear of death ${ }^{13}$ as well as general distress, anxiety, excessive alcohol consumption, and other psychiatric disorders. ${ }^{14}$ In Western Africa, the Ebola outbreak (2014-2015) devastated the health facilities and people's trust in health care providers, and as a result, a greater number of deaths are estimated to have resulted from childbirth, malaria and AIDS, and other diseases related to the absence or reduced access to non-Ebola services. ${ }^{15}$

\section{Shifting from a Response-driven to a Risk-driven Approach to $D D R$ in Health}

Disaster risk reduction encompasses the scientific, policy, and practice activities that aim to reduce losses in lives, livelihoods, and health. ${ }^{9}$ Ensuring emergency response to disasters is adequate and implements the highest standards for clinical and resource management to minimize injury, suffering, and loss of life, requires prevention and preparation across the emergency services environment across health and other sectors. ${ }^{16}$ Resilience - the bounce back factor - applies to both critical infrastructures, such as hospital buildings and transport systems, as well as organizations and their management according to the United Nations Office for Disaster Risk Reduction's (UNISDR; Geneva, Switzerland) Scientific and Technical Advisory Group (STAG). ${ }^{6}$ The World Health Organization (WHO: Geneva, Switzerland) has worked with member States to ensure hospitals and other health facilities are safe and fully operational before, during, and after disasters. ${ }^{17}$

The "Hospitals Safe from Disasters" campaign, supported by the WHO, the World Bank, and UNISDR, aimed at protecting the lives of patients, health staff, and the public better by reinforcing the structural resilience of health facilities and better preparing and training health workers on preparedness plans. ${ }^{18}$ The "One Million Safe Schools and Hospitals" initiative enabled the UNISDR Secretariat to work with communities, civil society organizations, governments, and the private sector to make schools and hospitals safe from disasters and has received over 200,000 pledges for safety. ${ }^{19}$ The Sendai Framework also promotes implementation in coordination with other relevant frameworks, such as the International Health Regulations, ${ }^{20}$ including through "the development and the strengthening of capacities and clear and focused programs that support the priorities of States in a balanced, well-coordinated, and sustainable manner, within their respective mandates."

Considering the diversity of sectors involved in successfully preventing mortality and morbidity from disasters, whole of government country assessments are of relevance. For example, during the implementation of the Hyogo Framework for Action, two countries (the UK and Finland) volunteered to be peer-reviewed by other countries to assess their performance on the implementation of the Hyogo Framework. ${ }^{21,22}$ The reviews showed that both countries had sophisticated emergency planning systems at local and national levels in place, and while health sector considerations didn't feature specifically, there was recognition that science underpinned good practice, particularly in the UK.

Finland's review commended its "whole-of-society approaches" and that its DRR policies:

Benefited also from a high level of trust among citizens, a cooperative attitude in society and a sense of solidarity, and high-quality public services from national to local levels. Furthermore, considerable use of research and information and communications technology (ICT), and the highly developed education system and social policies in Finland, contribute to an effective national risk management system which takes advantage of state-of-the-art innovations. ${ }^{22}$

\section{Discussion}

The Sendai Framework for DRR 2015-2030 and the Role of Health

Although there was little mention of health in the Hyogo Framework for Action 2005-2015, its implementation over the past decade has helped to widen the remit of DRR activities beyond simply responding to natural disasters and focusing on minimizing damage to infrastructure and economic activity. ${ }^{23}$ In the Hyogo Framework's successor, the Sendai Framework, health is an explicit outcome of DRR and the gamut of DRR activities has increased in breadth and sophistication to incorporate all-hazard risk assessment, impact-based forecasting, improving early warning and response capacities, better resource management, knowledge-creation and sharing, building public commitment and developing supportive institutional frameworks, and has contributed to shifting the culture from a hazard and responsedriven culture to a risk-driven, integrated culture that encompasses prevention, recovery, and rehabilitation. ${ }^{6,24}$

The Sendai Framework was adopted on March 18, 2015 by 187 United Nations (UN) Member States after extensive negotiations at the Third World Conference on Disaster Risk Reduction (Sendai, Japan). It is a voluntary agreement and a significant framework for health implementation and emphasizes 
the use of an all-hazards approach, linking to epidemics and pandemics and to the International Health Regulations. ${ }^{25}$ This far-reaching new Framework for DRR has a clear outcome, goal, seven global targets, and four priorities for action. The goal is to:

Prevent new and reduce existing disaster risk through the implementation of integrated and inclusive economic, structural, legal, social, health, cultural, educational, environmental, technological, political, and institutional measures that prevent and reduce hazard exposure and vulnerability to disaster, increase preparedness for response and recovery, and thus strengthen resilience.

Out of the seven global targets, the following five are particularly relevant to health: (1) substantially reduce global disaster mortality by 2030 , aiming to lower average per 100,000 global mortality between 2020-2030 compared to 2005-2015; (2) substantially reduce the number of affected people globally by 2030, aiming to lower the average global figure per 100,000 between 2020-2030 compared to 2005-2015; (3) substantially reduce disaster damage to critical infrastructure and disruption of basic services, among them health and educational facilities, including through developing their resilience by 2030; (4) substantially increase the number of countries with national and local DRR strategies by 2020; and (5) substantially increase the availability of and access to multi-hazard early warning.

The overall priorities for action of the Sendai Framework are:

- Priority 1: Understanding disaster risk;

- Priority 2: Strengthening disaster risk governance to manage disaster risk;

- Priority 3: Investing in disaster risk reduction for resilience; and

- Priority 4: Enhancing disaster preparedness for effective response and to "Build Back Better" in recovery, rehabilitation, and reconstruction.

The following paragraphs from the Sendai Framework include actions required by Public Health, which are agreed as priorities for WHO to act on in partnership with UNISDR and the UN System, as well as local, national, regional, and global partners, as relevant:

- In Priority 3: At National and Local Level 30(i) - Enhance the resilience of national health systems, including by integrating disaster risk management into primary, secondary, and tertiary health care, especially at the local level; developing the capacity of health workers in understanding disaster risk and applying and implementing DRR approaches in health work; promoting and enhancing the training capacities in the field of disaster medicine; and supporting and training community health groups in DRR approaches in health programs, in collaboration with other sectors, as well as in the implementation of the International Health Regulations (2005) of the WHO.

- In Priority 3: At National and Local Level 30(k) - People with life threatening and chronic disease, due to their particular needs, should be included in the design of policies and plans to manage their risks before, during, and after disasters, including having access to life-saving services.

- In Priority 3: At Global and Regional Level 31(e) - Enhance cooperation between health authorities and other relevant stakeholders to strengthen country capacity for disaster risk management for health, the implementation of the International Health Regulations (2005), and the building of resilient health systems.

- In Priority 4: At National and Local Level 33(c) - Promote the resilience of new and existing critical infrastructure, including water, transportation, and telecommunications infrastructure, educational facilities, hospitals, and other health facilities to ensure that they remain safe, effective, and operational during and after disasters in order to provide live-saving and essential services.

- In Priority 4: At National and Local Level 33(j) - Strengthen the design and implementation of inclusive policies and social safety-net mechanisms, including through community involvement, integrated with livelihood enhancement programs, and access to basic health care services, including maternal, newborn, and child health, sexual and reproductive health, food security and nutrition, housing, and education, towards the eradication of poverty, to find durable solutions in the post-disaster phase and to empower and assist people disproportionately affected by disasters.

- In Priority 4: At National and Local Level 33(n) - Establish a mechanism of case registry and a database of mortality caused by disaster in order to improve the prevention of morbidity and mortality.

- In Priority 4: At National and Local Level 33(o) - Enhance recovery schemes to provide psychosocial support and mental health services for all people in need.

Implementation of the Sendai Framework: What could be the Impact on the Health Sector?

The Sendai Framework gives a clear mandate emphasizing the need for a more integrative DRR processes, incorporating bottomup and top-down actions, local scientific and technical knowledge, and highlights synergies with health, climate change, and sustainable development which could support mutually beneficial capacity development and joint policy initiatives across these policy areas and policy sectors to enhance the mainstreaming of DRR in health. ${ }^{26}$

Working in partnership with the UNISDR STAG and linking health to DRR to implement the Sendai Framework could have significant impact, ${ }^{27}$ particularly when it has the following mandate in Priority 1 :

Para 25(g)- Enhance the scientific and technical work on disaster risk reduction and its mobilization through the coordination of existing networks and scientific research institutions at all levels and all regions with the support of the UNISDR Scientific and Technical Advisory Group in order to:

- strengthen the evidence-base in support of the implementation of this framework;

- promote scientific research of disaster risk patterns, causes, and effects;

- disseminate risk information with the best use of geospatial information technology;

- provide guidance on methodologies and standards for risk assessments, disaster risk modeling, and the use of data;

- identify research and technology gaps and set recommendations for research priority areas in DRR;

- promote and support the availability and application of science and technology to decision making; 


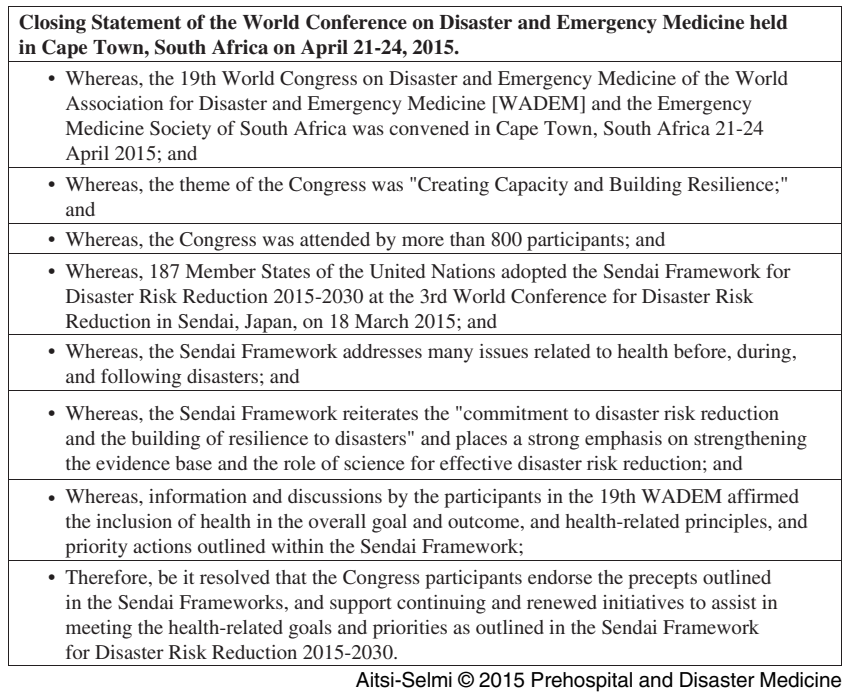

Figure 1. Closing Statement of the World Conference on Disaster and Emergency Medicine held in Cape Town, South Africa on April 21-24, 2015.

Abbreviation: WADEM, World Association for Disaster and Emergency Medicine.

- contribute to the update of the 2009 UNISDR Terminology on Disaster Risk Reduction;

- use post-disaster reviews as opportunities to enhance learning and public policy; and

- disseminate studies.

The need to communicate and understand the value of the framework widely so that all sectors, including health actors, embrace and implement the framework to protect people's health and well-being from the risks of disasters and poorly managed emergency response should be met by all, if progress on the health priorities is to be made. Crucially, an opportunity is present in the widening of health sector activities and remit from a health care focused, vertical systems approach to strengthening health systems resilience and collaborating with non-health sectors to influence the wider determinants of health. ${ }^{28}$

A number of implementation efforts by stakeholders in global DRR have been undertaken in the immediate wake of the Sendai Framework and include the World Conference on Disaster and

\section{References}

1. Aitsi-Selmi A, Egawa S, Sasaki H, Wannous C, Murray V. The Sendai Framework for Disaster Risk Reduction: renewing the global commitment to people's resilience, health, and well-being. Int J Disaster Risk Reduct. 2015;6(2): 164-176.

2. Ye L, Abe M. The impacts of natural disasters on global supply chains. ARTNeT Working Paper. Vol. 115. http://www.unescap.org/sites/default/files/AWP\%20No.\% 20115.pdf. Accessed January 4, 2015.

3. Rodriguez H, Wachtendorf T, Kendra J, Trainor J. A snapshot of the 2004 Indian Ocean tsunami: societal impacts and consequences. Disaster Prevention and Management: An International Journal. 2006;15(1):163-177.

4. World Health Assembly. Resolution 64.10: Strengthening national health emergency and disaster management capacities and resilience of health systems. http://apps. who.int/gb/ebwha/pdf_files/WHA64/A64_R10-en.pdf. Published 2011. Accessed June 19, 2015.

5. International Panel on Climate Change 2014. The IPCC Fifth Assessment Report: impacts, adaptation, and vulnerability (Working Group II). Published 2014 http://www.climatecouncil.org.au/uploads/cd929c5cfed40f6d7c508dd6c1f930cf.pdf. Accessed April 12, 2015.
Emergency Medicine held in Cape Town, South Africa on April 21-24, 2015, hosted by the World Association for Disaster and Emergency Medicine (WADEM; Madison, Wisconsin USA). Its closing statement concluded that the Congress participants endorsed the precepts outlined in the Sendai Framework, and support continuing and renewed initiatives to assist in meeting the health-related goals and priorities as outlined in the Sendai Framework $^{29}$ (Figure 1).

The Nepal Earthquake was a stark reminder of the potential devastation from natural disasters. The science and policy DRR community's call and endorsement for the need to strengthen the science-policy interface existed prior to the adoption of the Sendai Framework, ${ }^{30}$ but the Nepal Earthquake crystallized further the Sendai Framework call for the use of technology, partnership, and the dissemination of research. Innovative, knowledge sharing agencies, such as Evidence Aid (Oxford, United Kingdom), ${ }^{31}$ worked to provide much needed evidence-based rapid response resources to health and other humanitarian workers on the ground through partners in the region and international agencies, such as the UN and WHO efforts.

The World Association for Disaster and Emergency Medicine is well placed to encourage partnership across sectors and scientific disciplines for effective DRR through its mission to:

Disseminate scientific evidence and best practices in prehospital and emergency health care, public health, and disaster health and preparedness and provide academic and evidence-based input into the development/review of relevant policies and educational programs. ${ }^{32}$

\section{Conclusions}

The implementation phase of the Sendai Framework has begun, but it is only the first of three UN landmark agreements this year (the other two being the Sustainable Development Goals due in September 2015 and the climate change agreements due in December 2015). Here lies an opportunity to align the post-2015 DRR agenda with global public health needs of the $21^{\text {st }}$ Century through evidence-based policy and scientific activity mandated in the Sendai Framework. The Framework is a linchpin intended to help to integrate DRR within and across all sectors, including health, by aligning the implementation of DRR with other relevant health frameworks. If successful and monitored, the policy integration could result in measurable improvements in health outcomes from disasters in the next 15 years.

6. UNISDR Scientific and Technical Advisory Group (STAG). Science is used for disaster risk reduction: UNISDR Science and Technical Advisory Group report 2015. http://www. unisdr.org/we/inform/publications/42848. Published 2015. Accessed April 17, 2015.

7. UNISDR. Terminology for disaster risk reduction. Published 2009. http://www. unisdr.org/we/inform/publications/11586. Accessed July 31, 2015.

8. Murray V, Aitsi-Selmi A, Blanchard K. The role of public health within the United Nations post-2015 framework for disaster risk reduction. Int J Disaster Risk Reduct. 2015;6:28-37.

9. United Nations. General Assembly - 2015. Sendai Framework for Disaster Risk Reduction 2015-2030. http://www.preventionweb.net/files/resolutions/N1514318. pdf. Accessed June 19, 2015.

10. Natural Disasters \& Environmental Hazards in Centre of Disease Control - Yellow book Chapter 2: The Pre-Travel Consultation - Counseling \& Advice for Travelers. http://wwwnc.cdc.gov/travel/yellowbook/2014/chapter-2-the-pre-travel-consultation/ natural-disasters-and-environmental-hazards. Accessed April 6, 2015.

11. Ochi S, Hodgson S, Landeg O, Mayner L, Murray V. Disaster-driven evacuation and medication loss: a systematic literature review. PLOS Currents Disasters. 2014: 1. doi: 10.1371/currents.dis.fa417630b566a0c7dfdbf945910edd96. 
12. Egawa S. 2015. The second report of International Research Institute Disaster Science: fact finding mission to Philippines. http://irides.tohoku.ac.jp/media/files/ IRIDeS_Report_Haiyan_second_20150302.pdf. Accessed January 12, 2015.

13. Williams R, Drury J. Personal and collective psychosocial resilience: implications for children, young people, and their families involved in war and disasters. In: Cook D, Wall J, Cox P, (eds). Children, and Armed Conflict. Basingstoke, England and New York, USA: Palgrave McMillan; 2011.

14. Neria Y, Shultz JM. Mental health effects of Hurricane Sandy: characteristics, potential aftermath, and response. JAMA. 2012;308(24):2571-2572.

15. Walker PG, White MT, Griffin JT, Reynolds A, Ferguson NM, Ghani AC. Malaria morbidity and mortality in Ebola-affected countries caused by decreased health-care capacity, and the potential effect of mitigation strategies: a modeling analysis. Lancet Infect Dis pii: S1473-3099(15)70124-6. doi: 10.1016/S1473-3099(15)70124-6. [Epub ahead of print].

16. World Health Organization. Emergency and disaster risk management for health. World Health Organization Web site. http://www.who.int/hac/techguidance/ preparedness/en/. Accessed July 15, 2015.

17. World Health Organization. Comprehensive Safe Hospitals Framework. World Health Organization Web site. http://www.who.int/hac/techguidance/comprehensive_ safe_hospital_framework.pdf?ua=1. Accessed July, 312015.

18. United Nations. General Assembly - 2008 Annual Report of the Secretary General on the Implementation of the International Strategy for Disaster Reduction. http://www. unisdr.org/files/resolutions/N0850245.pdf . Accessed May 7, 2015.

19. United Nations. General Assembly - 2012 Annual Report of the Secretary General on the Implementation of the International Strategy for Disaster Reduction. http://www. unisdr.org/files/resolutions/A67335E.pdf. Accessed May 7, 2015.

20. World Health Organization. International Health Regulations (2005), 2nd edition. Geneva, Switzerland: World Health Organization; 2008; http://whqlibdoc.who.int/ publications/2008/9789241580410_eng.pdf. Accessed February 28, 2015.

21. UNISDR, EC, OECD. 2013 United Kingdom Peer Review - Building resilience to disasters: Implementation of the Hyogo Framework for Action (2005-2015). Published 2013. http://www.unisdr.org/files/32996_32996hfaukpeerreview20131. pdf. Accessed June 15, 2015.
22. UNISDR, EC, OECD. 2014 Finland Peer Review - Building resilience to disasters: Implementation of the Hyogo Framework for Action (2005-2015). Published 2014. http://www.unisdr.org/files/38523_20140717finlandpeerreport.pdf. Accessed June 15, 2015.

23. Helvetas Swiss Intercooperation. Disaster Risk Reduction \& Development: towards mainstreaming disaster risk reduction in development efforts. https://assets.helvetas. org/downloads/37_disasterriskreductionanddevelopmentdrr_global_red_inal_eng1_a4_ portrait.pdf. Accessed January 18, 2015.

24. International Federation of Red Cross and Red Crescent Societies (IFRCRCS). 2014 World Disasters Report: focus on culture and risk. http://www.ifrc.org/Global/ Documents/Secretariat/201410/WDR\%202014.pdf. Accessed January 21, 2015.

25. Aitsi-Selmi A, Murray V. The Sendai Framework: disaster risk reduction through a health lens. Bulletin of the World Health Organization. 2015;93:362.

26. World Health Organization. Health and Disaster Risk. A contribution by the United Nations to the consultation leading to the Third UN World Conference on Disaster Risk Reduction. http://www.wcdrr.org/uploads/HEALTH.pdf. Accessed April 12, 2015.

27. Carabine E. Revitalizing evidence-based policy for the Sendai Framework for Disaster Risk Reduction 2015-2030: lessons from existing international science partnerships. PLOS Currents Disasters. 2015;1. doi: 10.1371/currents.dis.aaab45b2b4106307ae2168a 485e03b8a. Accessed January 15, 2015.

28. World Health Organization. Commission on the Social Determinants of Health (CSDH) 2008. Closing the gap in a generation: health equity through action on the social determinants of health. Final Report of the Commission on Social Determinants of Health. http://whqlibdoc.who.int/publications/2008/9789241563703_eng_contents. pdf?ua=1. Accessed July 31, 2015.

29. World Association of Disaster and Emergency Medicine. Annual Conference 2015 Closing Statement. http://www.wadem.org/. Accessed May 12, 2015.

30. Calkins J. Moving forward after Sendai: how countries want to use science, evidence, and technology for disaster risk reduction. PLOS Currents Disasters. 2015;1. doi: 10.1371/currents.dis.22247d6293d4109d09794890bcda1878.

31. Evidence Aid Web site. http://www.evidenceaid.org/. Accessed August 8, 2015.

32. World Association of Disaster and Emergency Medicine. Vision. http://www.wadem. org/mission.html. Accessed June 17, 2015. 